\title{
Extremophiles: applications and roles in environmental sustainability
}

\author{
Naveen Kumar Arora ${ }^{1} \cdot$ Hovik Panosyan ${ }^{2}$
}

Published online: 31 July 2019

(c) Society for Environmental Sustainability 2019

Microorganisms are the most ubiquitous living entities on Earth. They are also the most diverse organisms, present in about every nook and corner of the blue planet. As per estimates only about $1 \%$ of the total microorganisms present on Earth have been discovered so far and there still are unexplored habitats where these omnipresent organisms may be present. Extremophilic microorganisms are a largely unexplored group that have the abilities to thrive in extreme conditions. These microbes have made useful adaptations in their genetic and metabolic machinery to thrive in the hostile conditions. Some of these microbes represent the very ancient life forms which must have thrived on Earth billions of years ago when the conditions were very different. In fact studying these microbes helps to find out how the evolution of life occurred on Earth and what type of conditions prevailed when life came on the planet.

Extremophiles are categorized according to conditions in which they grow. Thermophiles/hyperthermophiles can be found in habitats with high or very high temperatures such as hydrothermal vents, volcanic sites, hot springs; psychrophiles flourish in cold habitats such as polar regions, on the mountains at high altitudes, deep inside the oceans; barophiles which love high pressure conditions are mainly found deep inside the oceans or sea; acidophiles at $\mathrm{pH}$ less than 5.0 or even near to 0.0 , including habitats such as acid mine drainage sites, acidic lakes; alkalophiles thrive at highly alkaline $\mathrm{pH}$ such as in sodic lakes; halophiles love very high salt concentrations such as in sea, saline alkaline lakes; xerophiles can prosper in conditions with very low water availability which include deserts; metallophiles can tolerate and grow in presence of high concentrations of metals/heavy metals and there also are some other extremophilic

Naveen Kumar Arora

nkarora.bbau@gmail.com

1 Department of Environmental Science, Babasaheb Bhimrao Ambedkar University, Lucknow, UP 226025, India

2 Department of Biochemistry, Microbiology and Biotechnology, Yerevan State University, Yerevan, Armenia forms which can even survive and grow in presence of high concentration of nuclides, pollutants such as poly hydroxyl alkanoates (PAHs), pesticides etc., and can be termed as pollution loving microbes which have evolved mainly due to anthropogenic activities. Sometimes, multiple stresses are present in the niche simultaneously and extremophiles which are able to thrive in such habitats are defined as polyextremophiles (such as thermoacidophiles, haloalkaliphiles and so on). There are innumerous examples of these fascinating organisms which have been discovered now and these include primarily prokaryotes (bacteria and archaea) and some eukaryotes (algae, yeast and fungi). Hyperthermophilic archaea Pyrolobus fumarii and Geogemma barossii have been reported with ability to tolerate temperature up to $121{ }^{\circ} \mathrm{C}$. Another hyperthermophilic archaeon Methanopyrus kandleri strain 116 from deep sea hydrothermal field has been reported to withstand highest recorded temperature of $122{ }^{\circ} \mathrm{C}$ for any life form on Earth. Amongst psychrophiles, Psychrobacter cryopegellain isolated from Polar Regions of Siberian permafrost, Russia can survive up to $-20^{\circ} \mathrm{C}$. Thermophilic methanogens, Methanocaldococcus jannaschii and Methanothermococcus thermolithotrophicus are examples of barophilic microbes which have been isolated from high pressure niches of deep sea beds. Likewise, halophiles have been isolated from Great Salt Lake and Dead Sea. Halarsenatibacter silvermanii from a salt lake in USA is reported amongst the most halophilic microbe discovered and can survive in salt concentration of approximately $35 \%$. Thiobacillus, Sulfolobus and Thermoplasma are some common genera that are considered to be very acidophilic in nature. Recently, Picrophilusoshimae and Picrophilustorridus have been included as the most acidophilic archaea from hot spring in Noboribetsu, Japan, which can grow at $\mathrm{pH}$ as low as 0.06. Haloalkalophilic Halomonas campisalis reported from Soap Lake, USA can grow at $\mathrm{pH}$ up to 12. Metallophiles which are able to resist high concentrations of heavy metals such as cadmium, cobalt, copper, lead, mercury, nickel, zinc and so on, have been isolated from volcanic areas, geothermal and hydrothermal vents and from industrially polluted sites. Xerotolerant and radiation 
tolerant archeae, Halobacterium salinarum NRC-1 isolated from a salt mine is another important example of extremophilic microbe. The aforementioned are only a few striking examples of these amazing group of microbes and there are still myriad of possibilities of finding many novel species from the extreme habitats on Earth.

Extremophilic microorganisms not only are of ecological importance but are also gaining significance in biotechnological research and industrial uses. Thermophiles possess thermostable proteins and cell membranes that do not get denatured at elevated temperatures and resist proteolysis, whereas psychrophiles and barophiles have stable membranes, cell wall (at low temperatures and/or high pressure) and unique proteins; halophiles have increased concentration of inorganic ions and compatible solutes; acidophiles/alkaliphiles maintain their $\mathrm{pH}$ to neutral by pumping out excess ions by specific mechanisms. These microbes also maintain their membrane fluidity and stability under extreme conditions and protect their genetic system in such environment. This means extremophiles possess unique genes and know how to multiply under extreme conditions. Because of these characteristics extremophiles are unique and are now being extensively used for production of important biomolecules which are stable at high or low temperature, $\mathrm{pH}$ extremities, very high pressure and even in presence of deadly pollutants. These microbes or their metabolites are exceptional and can perform exclusive tasks in nature and at industrial level. For example they can provide stable enzymes at temperature and pressure extremes, can be used for biodegradation and bioremediation purposes in extreme habitats, source of biofuel and bioenergy, source of specialized pigments for solar cells able to work in extreme conditions such as polar caps and so on. Extremophilic microbes can thus play a very important role in future to achieve the targets of sustainability and biobased economy.

Extremozymes are already proving to be very important biomolecules for various industries. Till date, over 3000 enzymes have been isolated from different microbes and many of them are being successfully used in biotechnological and industrial processes but still there are so many resources which can be explored for novel biological metabolites. At present, compound annual growth rate (CAGR) of industrial enzymes at global level is $8.2 \%$ which is likely to grow in near future. With emerging advancements in biotechnological field, extremozyms can play a major role in future bio-based market growth. Expansion of industrial processes in light of novel extremozymes is going to be of utmost importance; particularly with changing scenario of climate and increasing level and diversity of pollutants. Innovative techniques will also be required to overcome bottlenecks related to uncultured extremophiles and obtaining the metabolites/enzymes from them at large scale. Thermostable DNA polymerases used in polymerase chain reactions (PCR), are among the best examples of industrial application of thermostable enzymes. Thermophiles such as Thermus aquaticus (Taq), Thermococcus litoralis (Vent) and Pyrococcus furiosus (Pfu) are key players in PCR-based diagnostics. Variety of hydrolases e.g. amylases, cellulases, esterases, lipases, peptidases and xylanases have been isolated from various extremophiles (psychrophiles, halophiles, alakalophiles/acidophiles) offering great industrial and biotechnological potential, for example, in detergents, petroleum, pulp and paper industries, in food processing and beverage industries and for bioremediation purposes. Cold active enzymes from psychrophiles have their own benefits as they possess high catalytic activity at very low temperatures due to greater flexibility of their proteins. Moreover, these require no expensive heating and give high reaction yields even in cold conditions which reduces the energy consumption. Certain pressure related proteins are being used from barophiles for production and sterilization of food items at varied pressure conditions. With help of genetic and chemical manipulations, the productivity, efficiency and stability of these enzymes can be improved for industrial processes.

Apart from industrial benefits research on extremophiles can be useful to understand the functioning of extreme ecological systems. Over the last few years scientists and researchers have been fascinated by the microbial life present in extreme habitats. The information of microbial composition and biogeochemical cycling of biogenic elements of such ecosystems would help to understand global change, threats and opportunities posed by the life in these habitats. Biotechnological tools can further help in identifying and utilizing these amazing microbes in tackling issues such as restoration of polluted ecosystems, provide increased yields of degraded habitats etc. There is still a huge diversity of microorganisms which is waiting to be explored particularly from habitats untouched by humans, hence many useful species and metabolites will definitely be discovered and utilized for improving the quality of life. This special issue explores different aspects of extremophiles including mechanisms of their survival and multiplication in challenging environmental conditions particularly imposed by anthropogenic activities. Extreme ecosystems are a unique biological resource and hot bed of genetic diversity so it is important to mine this wealth for various green purposes. The topics in the issue include but are not limited to: diversity and microbial ecology, physiology, genetic systems, microbe-environment interactions, adaptation and evolution, element cycling and biotechnological applications of microbes in changing and extreme ecosystems. In near future these incredible creatures are going to play a very crucial role in maintaining the environmental sustainability.

Publisher's Note Springer Nature remains neutral with regard to jurisdictional claims in published maps and institutional affiliations. 